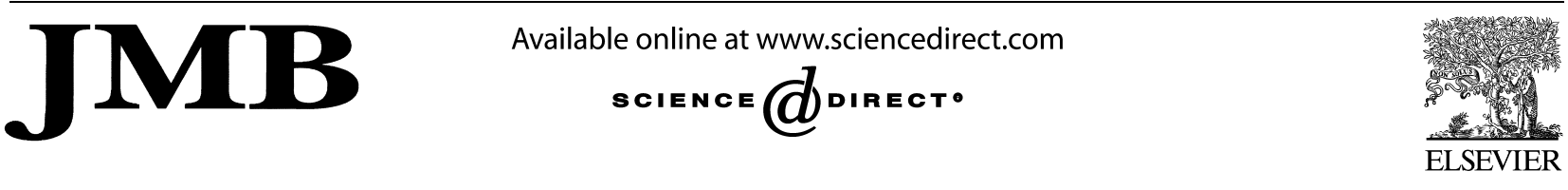

\title{
The Mechanism of Proton Exclusion in the Aquaporin-1 Water Channel
}

\section{Bert L. de Groot ${ }^{1 *}$, Tomaso Frigato ${ }^{2}$, Volkhard Helms ${ }^{2}$ and Helmut Grubmüller ${ }^{1}$}

\author{
${ }^{1}$ Theoretical Molecular \\ Biophysics Group \\ Max-Planck-Institute for \\ Biophysical Chemistry, Am \\ Fassberg 11, 37077 Göttingen \\ Germany \\ ${ }^{2}$ Theoretical Biophysics Group \\ Max-Planck-Institute for \\ Biophysics, Kennedyallee 70 \\ 60596 Frankfurt am Main \\ Germany
}

\begin{abstract}
Aquaporins are efficient, yet strictly selective water channels. Remarkably, proton permeation is fully blocked, in contrast to most other water-filled pores which are known to conduct protons well. Blocking of protons by aquaporins is essential to maintain the electrochemical gradient across cellular and subcellular membranes. We studied the mechanism of proton exclusion in aquaporin-1 by multiple non-equilibrium molecular dynamics simulations that also allow proton transfer reactions. From the simulations, an effective free energy profile for the proton motion along the channel was determined with a maximum-likelihood approach. The results indicate that the main barrier is not, as had previously been speculated, caused by the interruption of the hydrogen-bonded water chain, but rather by an electrostatic field centered around the fingerprint Asn-Pro-Ala (NPA) motif. Hydrogen bond interruption only forms a secondary barrier located at the ar/R constriction region. The calculated main barrier height of $25-30 \mathrm{~kJ} \mathrm{~mol}^{-1}$ matches the barrier height for the passage of protons across pure lipid bilayers and, therefore, suffices to prevent major leakage of protons through aquaporins. Conventional molecular dynamics simulations additionally showed that negatively charged hydroxide ions are prevented from being trapped within the NPA region by two adjacent electrostatic barriers of opposite polarity.
\end{abstract}

(C) 2003 Elsevier Ltd. All rights reserved.

Keywords: Q-HOP; proton transfer; molecular dynamics simulation; proton gradient; membrane permeability for the cell to maintain the water balance under different conditions, and thus to allow water molecules to efficiently permeate their bilayer membranes. Specialized channels, so-called aquaporins, have therefore evolved for that purpose. ${ }^{2}$

Aquaporins constitute a large and ubiquitous family of integral membrane proteins that facilitate highly efficient, yet strictly selective passive permeation of water and other small neutral solutes across biological membranes ${ }^{3-7}$ by allowing water molecules to form hydrogen-bonded chains through their channels. Despite the fact that hydrogen-bonded water chains are generally known to conduct protons well, ${ }^{8-11}$ aquaporins are unusual water-filled pores, in that they restrict proton flux through the pore to a rate that is at least 1000-fold slower than the water flux. Moreover, not only the hopping of protons between water molecules in the pore region should be hindered, but also the co-diffusion of hydronium (or the negatively charged hydroxide) ions together with water

Abbreviations used: MD, molecular dynamics; bAQP1, bovine aquaporin-1.

E-mail address of the corresponding author: bgroot@gwdg.de 
molecules must be prevented. The question of how aquaporins prevent proton permeation through their water-filled pores, although controversially discussed for many years, is still unresolved.

Almost 200 years ago, von Grotthuss proposed the idea of "conducting wires" in water. ${ }^{12}$ Although he was not aware of the underlying mechanism that caused the conductance of water, the basic concept later proved to be valid, when further refinement of the mechanism became feasible through experiments and quantum chemical calculations. ${ }^{10,13,14}$ In bulk water, protons can efficiently "hop" from one water molecule to the next along a network of hydrogen bonds. After each proton transfer, the changed electrostatic charge distribution forces the involved water molecules to rearrange and thus to optimize the local hydrogen network, which would suggest that a reduced rotational water mobility hinders proton conduction. However, also in other water conducting pores, like the model pore gramicidin A, proton conduction along a chain of water molecules has been observed, ${ }^{15}$ despite considerable restriction of the rotation of water molecules. ${ }^{8,9}$ In carbon nanotubes, an even larger proton hopping rate along one-dimensional water chains than in bulk water has been described. ${ }^{16}$ Protonconducting water chains also underlie the function of the proton pump bacteriorhodopsin. ${ }^{17-20}$ Similarly, proton leakage across biological lipid bilayer membranes has been proposed to occur via transient water chains. ${ }^{21,22}$

In humans alone, more than ten different aquaporins with specialized functionality are known and expressed in tissues as diverse as kidney, red blood cells, and the brain. A number of severe diseases have been identified that are caused by dysfunctional aquaporins..$^{5,23,24}$ Not only the waterspecific aquaporin channels belong to the aquaglyceroporin superfamily, but also glycerol facilitators, like the bacterial glycerol facilitator GlpF. $^{25}$ Structural analyses of human ${ }^{26,27}$ and bovine ${ }^{28}$ aquaporin- 1 as well as of the homologous $\mathrm{GlpF}^{29}$ provided the first insight into the mechanism of solute permeation through their pores. Aquaglyceroporins fold into homo-tetramers of independent monomeric channels, ${ }^{30,31}$ with each monomer consisting of six transmembrane helices (Figure 1(a)). Two highly conserved loops, each containing the fingerprint Asn-Pro-Ala (NPA) motif, fold back into the protein and meet in the center of the channel. ${ }^{32}$ These loops leave the channel on either side, each forming a short $\alpha$-helix ${ }^{33}$ (helices B and E). The positive sides of the macro-dipoles associated with these short helices ${ }^{34}$ meet in the center of the channel. At this position, the side-chains of both asparagine residues of the NPA motifs have been proposed to isolate a passing water molecule from its neighbors by exclusively forming hydrogen bonds to the water oxygen, thereby putatively impeding the hopping of a proton to and from this water molecule. ${ }^{26}$

Other indirect evidence on the mechanism of proton blockage in aquaglyceroporins came from conventional molecular dynamics (MD) simulations of water permeation through the aquaporin-1 and GlpF pores. ${ }^{35,36}$ We have found that water molecules are strongly oriented in the pore, and that the hydrogen-bonded water network is severely interrupted within the ar/R constriction region of the pore which is located approximately $10 \AA$ towards the extracellular side from the NPA region, and have proposed that this interruption forms a proton barrier. ${ }^{35}$ In MD simulations of GlpF, strong alignment of water molecules was also observed. ${ }^{35,36}$ Accordingly, this alignment was found to be weakened when the charges on the B and E helices were switched off. ${ }^{36}$ The local orientational restriction of water molecules in the NPA region was proposed to play a major role in the proton exclusion mechanism in aquaglyceroporins. ${ }^{36}$ Although the details of the proposed mechanisms differ, they have in common that an interruption of the hydrogen-bonded water chain, either at the ar/ $\mathrm{R}$ constriction site or at the NPA motif, forms the main barrier for protons to traverse across the channel.

However, all proton exclusion mechanisms that have been proposed so far are based on indirect evidence only, either on detailed analyses of the aquaporin structure, or on the behavior of water molecules in the aquaporin pore during conventional force field based MD simulations, which cannot describe intrinsically quantum mechanical transfer reactions of protons. Here, we report a direct approach by analyzing the dynamics of protons in the pore using both Q-HOP ${ }^{37}$ molecular dynamics simulations, which explicitly describe the intrinsically quantum mechanical proton transfer processes, as well as conventional force field-based MD simulations to study the diffusion of $\mathrm{H}_{3} \mathrm{O}^{+}$ions. Finally, because the permeation of a hydroxyl $\left(\mathrm{OH}^{-}\right)$ion across the membrane in one direction effectively translocates a proton in the opposite direction, we have also analyzed the dynamics of hydroxyl ions in the aquaporin-1 pore.

\section{Results and Discussion}

\section{Behavior of protons, $\mathrm{H}_{3} \mathrm{O}^{+}$and $\mathrm{OH}^{-}$in the pore}

As described in detail in Methods, the dynamic behaviour of aquaporin monomers or tetramers with inserted protons, $\mathrm{H}_{3} \mathrm{O}^{+}$and $\mathrm{OH}^{-}$was characterized extensively using MD simulations. We first present the main findings of the simulations and their biological relevance.

Figure 2 shows the motion of protons along the pore direction in Q-HOP MD simulations that started from configurations with protons at different positions in the pore. As can be seen, protons initially placed near the center of the pore in the vicinity of the NPA region quickly start leaving the pore in either the extracellular (upwards) or intracellular (downwards) direction. Towards the 

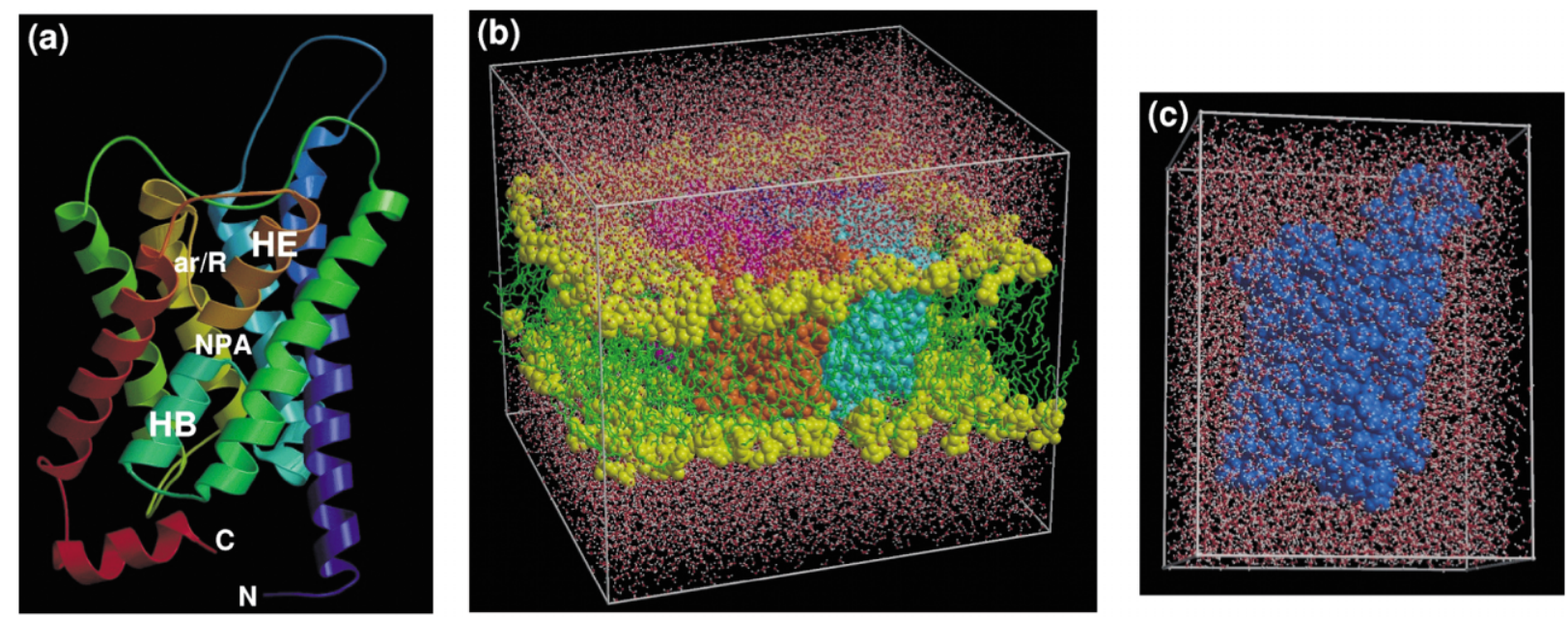

Figure 1. (a) The monomeric structure of bovine AQP1, showing the six-transmembrane helices and the short helices formed by loops $\mathrm{B}$ and $\mathrm{E}$ (HB and HE) that both contain the fingerprint NPA motif, which meet in the center of the pore. The ar/R constriction region is located $10 \mathrm{~A}$ extracellular from the NPA region. The simulation systems for the conventional MD simulations, in which an AQP1 tetramer was embedded in a solvated POPE bilayer, and for the Q-HOP simulations, in which an AQP1 monomer in water was simulated are shown in (b) and (c), respectively.

intracellular side, protons reach the channel entry/ exit rather efficiently, whereas those that travel towards the extracellular side typically get stuck for some time at the ar/R constriction region before they leave the channel (see also Figure 3). Eventually, all protons that started inside the pore leave the channel on either side. Few protons cross the NPA region during passage; most leave the NPA region at the same side at which they were placed initially. All crossings of the ar/R constriction region are from the center of the chan- nel (NPA) towards the extracellular side. No hops were observed that resulted in a translocation of a proton from the extracellular side of the channel towards the NPA region. These findings clearly indicate a strong barrier for proton conduction, located near the NPA region, as well as a smaller one located near the ar/R constriction region.

Conventional MD simulations including excess $\mathrm{H}_{3} \mathrm{O}^{+}$phenomenologically show the same behavior as the proton hopping Q-HOP simulations, albeit at a much lower rate (Figure 4, top).

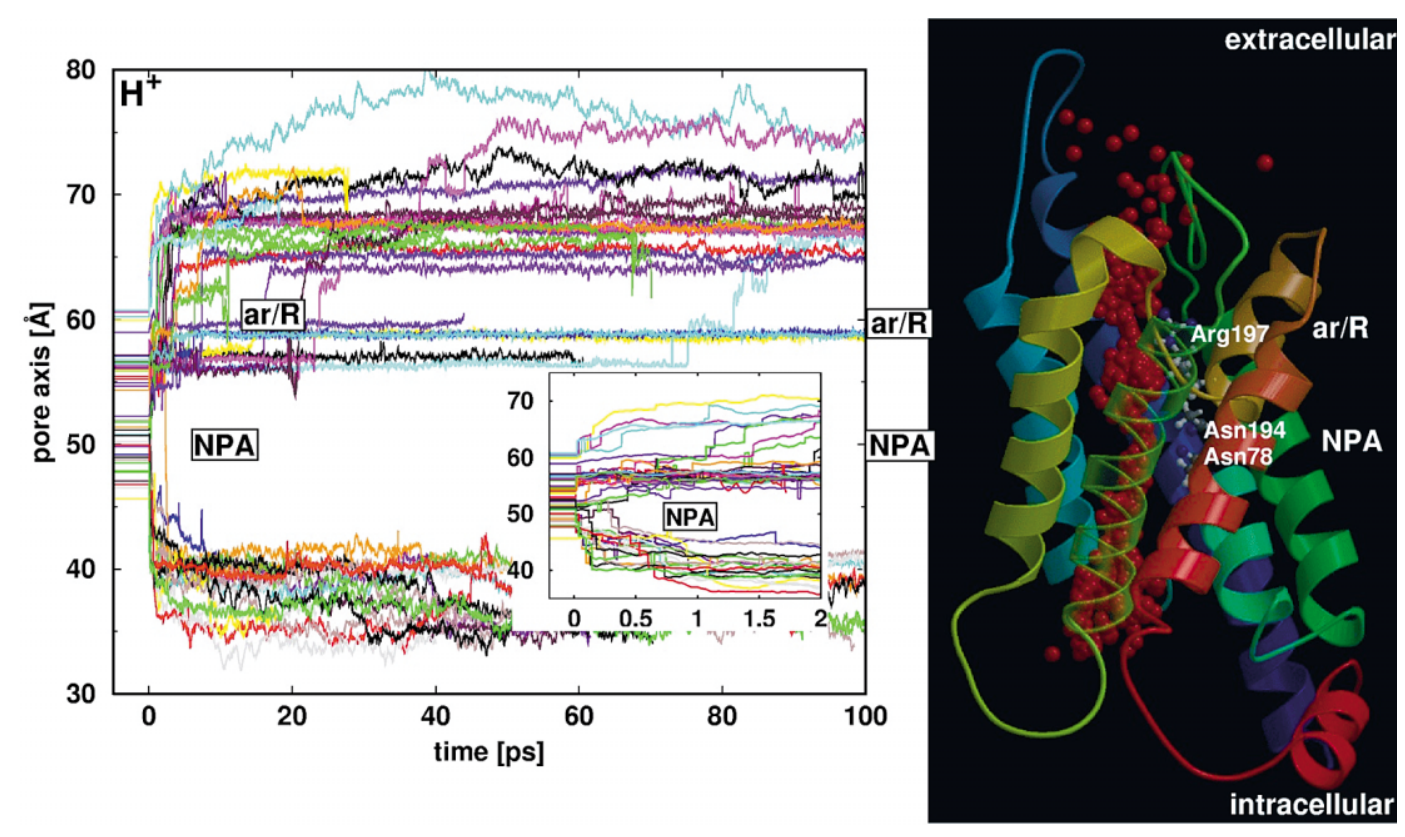

Figure 2. Motion of individual protons along the pore axis during all Q-HOP MD simulations (left). The protons' starting positions for the 48 simulations are marked at the left (horizontal line sections). To facilitate structural interpretation of the pore axis coordinate, the bAQP1 X-ray structure is shown, together with all proton positions at which a hop was observed (right). 

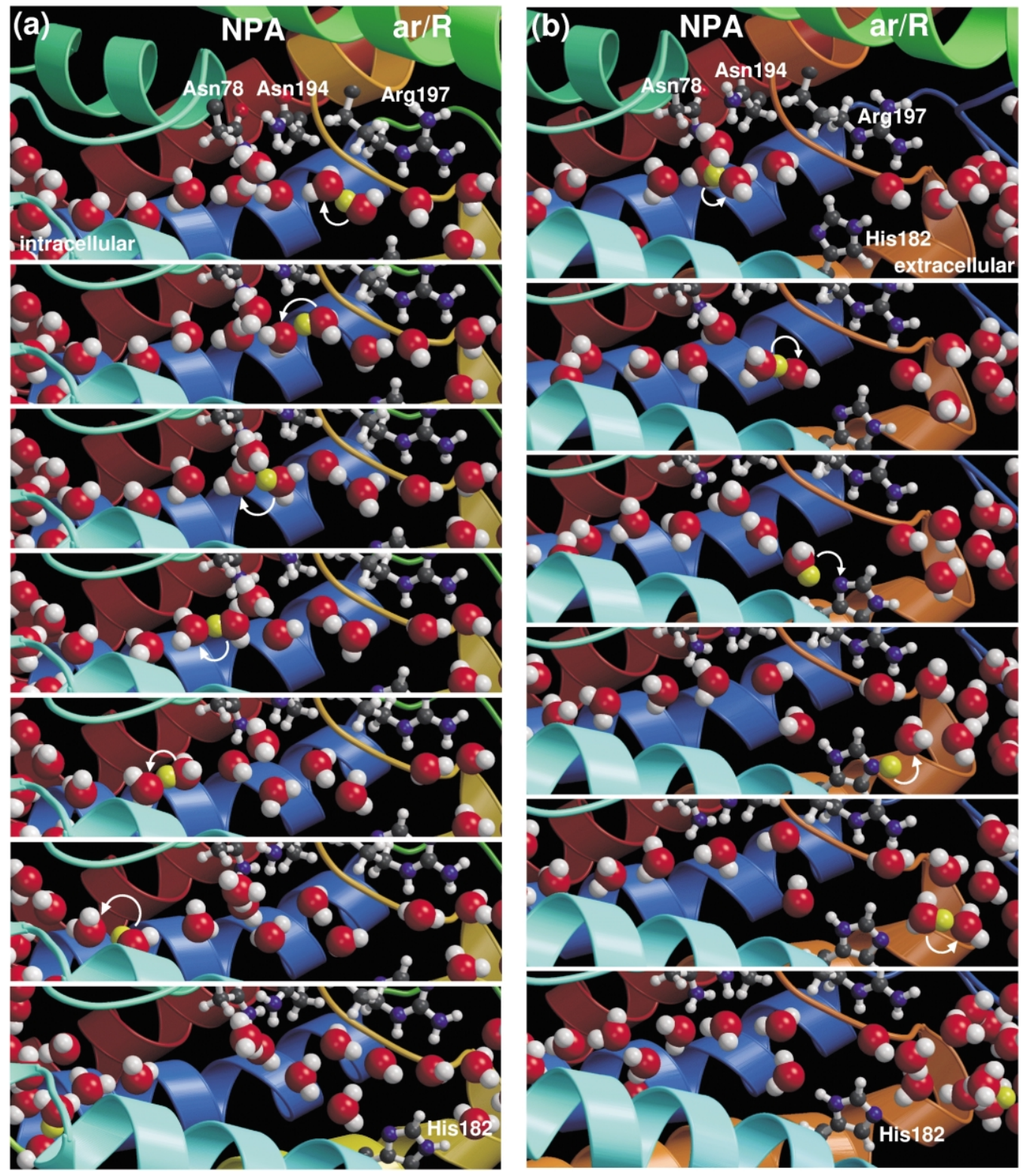

Figure 3. Snapshots of Q-HOP simulations during hop events along a typical path of a proton on its way out of the AQP1 pore towards (a) the intracellular side and (b) the extracellular side.

Whereas in the Q-HOP simulations, protons typically leave the pore within $1 \mathrm{ps}, \mathrm{H}_{3} \mathrm{O}^{+}$take about 20 ps. Despite this difference in time-scales, the barrier seems to be capable of blocking both proton transfer reaction and diffusion of $\mathrm{H}_{3} \mathrm{O}^{+}$(to discriminate between these two cases, we will subsequently use the term proton to refer to the first, and $\mathrm{H}_{3} \mathrm{O}^{+}$or hydronium ion to refer to the latter proton motion process). However, although proton hops across the ar/ $\mathrm{R}$ constriction region are observed (Figure 2), no diffusion of $\mathrm{H}_{3} \mathrm{O}^{+}$across this barrier is seen (Figure 4, top), an observation that also has been made for the M2 channel of the influenza A virus. ${ }^{38}$

One must expect that the proton barrier acts as a sink for the oppositely charged hydroxide $\left(\mathrm{OH}^{-}\right)$ ions, which therefore would block water permeation through the pore. Indeed, Figure 4 (bottom panel) shows that excess $\mathrm{OH}^{-}$display a behavior nearly opposite to that of the positive charges. In particular, those $\mathrm{OH}^{-}$that started from positions near the NPA region are attracted towards the NPA region and are trapped there. However, those $\mathrm{OH}^{-}$that were started farther away from the center of the pore (which is the physiologically relevant situation) either avoid the pore region or are expelled from the pore just like protons. This observation suggests secondary barriers for hydroxide ions at both sides of the main NPA proton barrier.

Using the maximum likelihood approach described in the Appendix, we constructed an effective free energy profile for the proton as a function of the pore axis from the collection of non-equilibrium Q-HOP simulations with an excess proton. The obtained profile (Figure 5) 


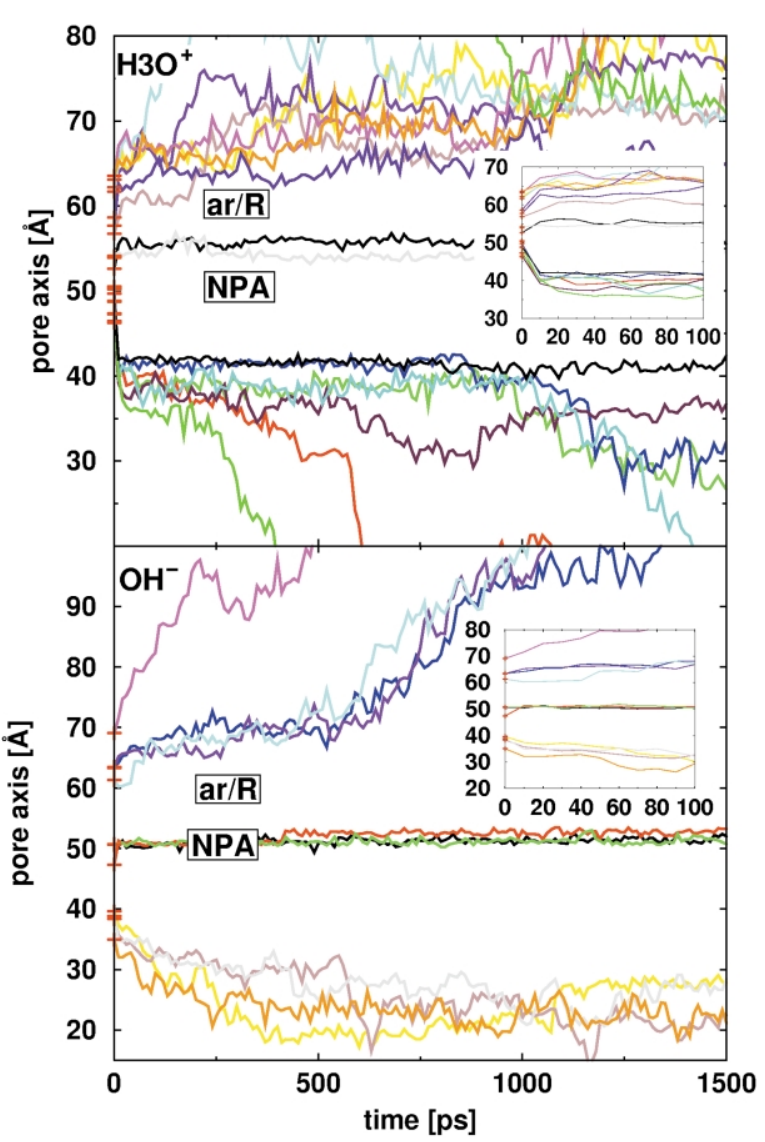

Figure 4. Diffusion of $\mathrm{H}_{3} \mathrm{O}^{+}$(top) and $\mathrm{OH}^{-}$(bottom) as observed in conventional molecular dynamics simulations. The initial positions are marked in red.

shows a clear maximum near the NPA region, as was expected from the observed motion of protons within the pore. Also the rest of the profile matches the observed qualitative behavior of protons in the pore. Closer inspection reveals a secondary barrier near the ar/ $\mathrm{R}$ constriction region and two minima at both the intracellular and extracellular sides of the pore center. As can also be seen in Figure 5, the non-equilibrium distributions of both the protons that were allowed to undergo quantum mechanical proton transfer reactions, as well as those described by diffusion of $\mathrm{H}_{3} \mathrm{O}^{+}$, qualitatively match the free energy profile. Note that a similar qualitative match is seen for the $\mathrm{OH}^{-}$between (the negated) free energy profile and their nonequilibrium distribution.

Near the free energy barrier at the NPA region, relatively low proton and $\mathrm{H}_{3} \mathrm{O}^{+}$densities are observed whereas relatively high densities are observed in the regions with lower free energy (around $40 \AA$ and $70 \AA$, respectively). The density profile for hydroxide ions is largely complementary to that of protons and $\mathrm{H}_{3} \mathrm{O}^{+}$. Highest hydroxide densities are observed near the NPA region and near the intracellular face of the pore, whereas relatively low densities are observed near the proton free energy minima near $40 \AA$ and $70 \AA$. Note that the high proton and $\mathrm{H}_{3} \mathrm{O}^{+}$densities near the ar/ $\mathrm{R}$ constriction region are non-equilibrium effects, reflecting protons and $\mathrm{H}_{3} \mathrm{O}^{+}$that started in the pore near the NPA region and got stuck in the ar/ $R$ region before being able to leave the channel on the extracellular side. The equilibrium density of protons and $\mathrm{H}_{3} \mathrm{O}^{+}$can therefore be expected to be much lower in this region, which is consistent with the free energy profile, where the minimum just intracellular from the ar/ $\mathrm{R}$ region is only a secondary one.

The proton minima near $40 \AA$ and $70 \AA$ are only transiently occupied by protons and $\mathrm{H}_{3} \mathrm{O}^{+}$. These wells are too shallow to trap a proton (or $\mathrm{H}_{3} \mathrm{O}^{+}$) tightly and, as can be seen from the longer conventional MD simulations (Figure 4, top), $\mathrm{H}_{3} \mathrm{O}^{+}$indeed leave these wells on both sides at intermediate timescales. Note that, because of the relatively low bulk proton concentration at physiological $\mathrm{pH}$, there is an entropic cost due to the confinement of the proton's available configurational volume to part of the aquaporin pore as compared to the

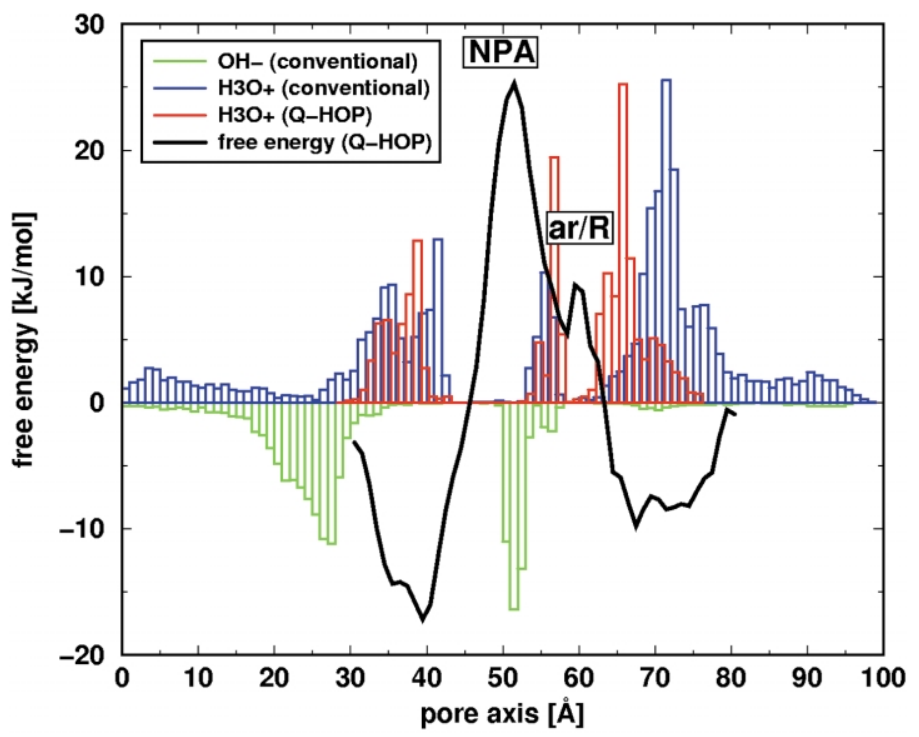

Figure 5. Maximum likelihood free energy profile for protons along the pore axis in Q-HOP simulations (black curve). For comparison, the relative non-equilibrium distributions of protons (red), $\mathrm{H}_{3} \mathrm{O}^{+}$ ions (blue), and $\mathrm{OH}^{-}$(green) are shown. 
mean volume available in bulk water, which explains why protons are not trapped within these minima.

\section{Structural determinants of proton exclusion}

The location of the main barrier for protons and $\mathrm{H}_{3} \mathrm{O}^{+}$near the NPA region is somewhat unexpected, because the interruption of the hydrogen bonded water network inside the pore, which was generally assumed to form the main barrier for protons, is most pronounced within the ar/R constriction region ${ }^{35}$ (see also Figure 6(b)). Also a previously proposed alternative mechanism

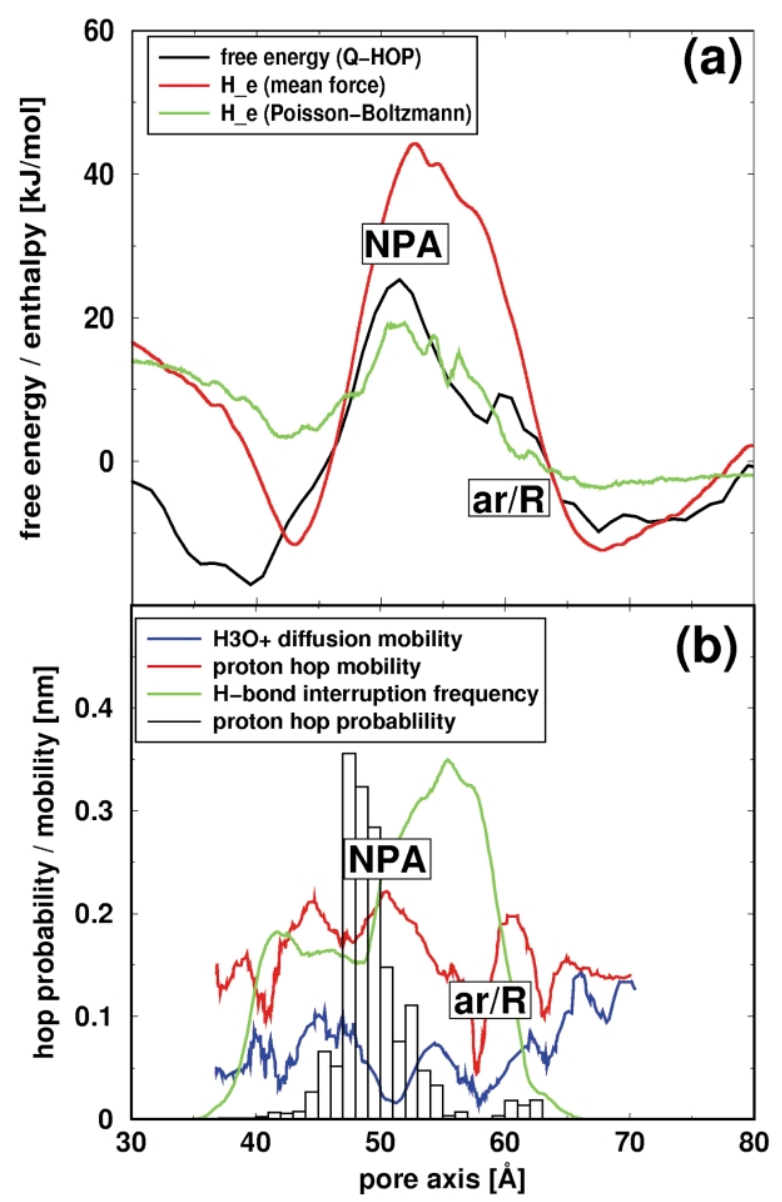

Figure 6. Determinants of proton exclusion. (a) The effective electrostatic potential for a proton inside the pore, based on the mean force on a probe charge (red) together with the maximum likelihood proton free energy profile (black), and the continuum PoissonBoltzmann electrostatic profile (green). (b) The role of hydrogen bond intactness between water molecules on the hopping efficiency in Q-HOP simulations. Shown are hop probabilities (for a Q-HOP to a neighboring water molecule) as a function of the pore axis (black) and the distances traveled along the pore direction due to proton hopping events (red) and $\mathrm{H}_{3} \mathrm{O}^{+}$diffusion (blue). For comparison, the frequency of interruption of contiguous hydrogen bonded water chains as observed in a conventional MD simulation of human AQP1 ${ }^{35}$ is shown in green. involving a local isolation of the water oxygen atom by both asparagine residues of the NPA motifs, which would thus prevent a proton from hopping onto this water molecule, ${ }^{26}$ now seems unlikely, since no such exclusive isolation of water molecules from other water molecules was observed in our simulations. Moreover, a recent refinement of this mechanism, ${ }^{36}$ involving an additional barrier caused by the putative reorientation step of the Grotthuss mechanism against the local electrostatic field also seems unlikely, since we actually more frequently observed proton hops in this region than in others (cf. Figure 6(b)). Even a number of hop events across the NPA region are observed.

This finding puts even more relevance on the question of what are the underlying structural and energetic determinants that cause proton exclusion and the main proton barrier in the NPA region as well as the secondary barrier in the ar/R constriction region. In particular, one would like to know to what extent the barrier is electrostatic in nature, as opposed to being caused by interruption of hydrogen-bonded water chains.

A comparison of the proton free energy profile to an estimate for the mean electrostatic potential in the pore (Figure 6(a)) shows a qualitative correspondence between the two profiles. In particular, the location of the main maximum agrees very well, as does the presence of the two minima. Not seen in the mean electrostatic potential profile is the secondary maximum, and also the location of the two minima appears to be somewhat shifted. This qualitative agreement suggests that the main determinants for the proton exclusion mechanism in aquaporins are electrostatic interactions. The most prominent electrostatic features of the pore are the macro-dipoles of the B and E loop helices formed by their positive N-terminal ends, which meet in the NPA region in the central part of the pore. These results suggest that the strong electric field blocks proton permeation not indirectly by perturbation of the Grotthuss mechanism, but rather mainly directly by creating an electrostatic barrier for protons as well as for $\mathrm{H}_{3} \mathrm{O}^{+}$and other cations.

As can be seen in Figure 6(a), the electrostatic potential estimate monotonously decreases from the NPA region towards the extracellular side of the channel, and can therefore not explain the presence of the secondary maximum in the proton free energy profile near the ar/R constriction region. The contiguous decrease of the electrostatic potential within the ar/ $\mathrm{R}$ region is unexpected, because the side-chain of a positively charged arginine residue (Arg197 for bAQP1, Arg195 for human AQP1) faces the pore in this region. Interestingly, this is the region where a frequent interruption of the hydrogen-bonded water network was previously observed which was predicted to disfavor proton hopping events between water molecules. ${ }^{35}$ Indeed, when the hop probability to neighboring water molecules is calculated as a 
function of the pore position, the ar/ $\mathrm{R}$ constriction region displays an extremely low hop probability of less than $0.01 \%$, whereas near the NPA region, values up to $30 \%$ are observed (cf. Figure $6(\mathrm{~b})$ ). Moreover, 15 of the 20 proton trajectories that ended at the extracellular side, hopped via His182 in the ar/ $\mathrm{R}$ region due to the lack of interwater hydrogen bonds in this region (see also Figure 3). These observations strongly suggest the secondary, local maximum in the ar/ $R$ constriction region to be caused by disruption of the hydrogen bond network between water molecules.

Additionally, the ar/ $\mathrm{R}$ constriction region also forms a barrier for $\mathrm{H}_{3} \mathrm{O}^{+}$(Figure 4, top panel and Figure 6(b)). An analysis of hydrogen bond enthalpies of water molecules in the pore showed a maximum in the hydrogen bond energy among water molecules in this region. ${ }^{35}$ This indicates that also the $\mathrm{H}_{3} \mathrm{O}^{+}$barrier in this region is hydrogen-bond mediated.

Note that the hop probabilities shown in Figure 6(b) not only result from the local intactness of the hydrogen bonded water chain, but may also be affected by an uneven distribution of reorientation energies connected to the turn of water molecules that follows a hop in the Grotthuss ${ }^{12}$ or "hop-and-turn" mechanism"1 to optimally align their dipoles with the new charge distribution. Unfavorable alignment of water dipoles with the macro-dipoles of the B and E helices in the NPA region during such reorientation events had previously been proposed to form the main proton exclusion barrier in aquaporins. ${ }^{36}$ The fact that hop events are frequently observed in the NPA region suggests, however, that the reorientation barriers exist in this region are generally small and, therefore, are not a main determinant for proton blockage.

Our prediction that electrostatic effects dominate the mechanism of proton exclusion could be tested by a number of experimental techniques. First, point mutations near the NPA region that would reduce the positive electrostatic potential locally are expected to reduce the barrier for protons to pass the pore, e.g. be Phe24Asp or Phe24Glu. Phe24 is located across the channel from both Asn residues from the NPA motif. A negatively charged residue at this location can be expected to reduce the local positive electrostatic potential caused by the macro-dipoles of the $B$ and E helices. Second, with a voltage clamp experiment it might be possible to measure the voltage dependence of proton conduction across a membrane with embedded aquaporins, in comparison to pure lipid membranes, which could yield information on the electrostatic barrier width.

\section{The barrier height}

The estimated barrier height for protons through the AQP1 pore is approximately $25-30 \mathrm{~kJ} \mathrm{~mol}^{-1}$
(Figure 5). Is this barrier high enough to prevent protons from leaking into the cell, which would destroy the electro-chemical gradient across the membrane? In this respect, it should be noted that the experimentally determined proton permeabilities of phospholipid bilayers ${ }^{39-42}$ range from $1 \times 10^{-5}$ to $5 \times 10^{-4} \mathrm{~cm} \mathrm{~s}^{-1}$, and thus are only slightly lower than permeability values typically reported for water. ${ }^{43,44}$ Since the estimated barrier height for protons through the AQP1 pore of approximately $25-30 \mathrm{~kJ} \mathrm{~mol}^{-1}$ is very close to the barrier height of typical membranes for water, ${ }^{45}$ the permeability of AQP1 for protons can be expected to be in the same range as the water and proton permeabilities of lipid membranes. Apparently, aquaporins, or any other membrane protein, need not have a lower proton permeability than the intrinsic membrane permeability for protons.

Assuming a $\mathrm{pH}$ gradient of $0.75 \mathrm{pH}$ unit (the typical proton gradient across the mitochondrial membrane during respiring conditions) and a membrane potential of $170 \mathrm{mV}$, the free energy required to pump a proton across the membrane (or that is released by proton diffusion in the opposite direction) is approximately $20 \mathrm{~kJ} \mathrm{~mol}^{-1}$, which in a simple approximation would correspond to a shift of $+10 \mathrm{~kJ} \mathrm{~mol}^{-1}$ on one side and of $-10 \mathrm{~kJ} \mathrm{~mol}^{-1}$ on the other side of the membrane in the bulk regions of the free energy profile (Figure 5). Adequate blocking of protons, therefore, can be expected also in this situation.

From an evolutionary viewpoint it is interesting to note that, apparently, the proton barrier is not larger than absolutely necessary. This suggests that a compromise between efficient water channels and proton filters has been achieved during evolution, resulting from a tradeoff between, on one hand, the optimization of the water permeability and, on the other hand, the blocking of protons and other ions.

Errors in the barrier height, as estimated from the effective maximum-likelihood free energy profile (Figure 5, see also Appendix), might arise from limited statistics (number of hops near the top of the barrier) and from the assumption that the observed hop rates are proportional to the Boltzmann factor of the free energy difference between adjacent slices in the pore. The latter rests on the assumption that the proton's vicinity is close to equilibrium at all times, as is also generally assumed in Kramers' theory. However, although we do expect fast equilibration to occur, it may in some instances not be fast enough in light of the partially fast motion of the proton. In that sense, the obtained energy profile should not be interpreted as an equilibrium free energy, but rather as one that is specifically adapted to the time-scale set by the motion of the proton. Since this motion, as the process of interest, dictates the relevant time-scale, we feel that our non-equilibrium approach is more appropriate than a full equilibrium treatment would be. 


\section{Hydroxide exclusion}

Like protons, $\mathrm{OH}^{-}$also must be excluded from the pores of aquaglyceroporins, since permeation of hydroxide ions in one direction would be equivalent to the leakage of a proton in the opposite direction. Furthermore, the proton main electrostatic barrier at the NPA region is a deep well for $\mathrm{OH}^{-}$or other anions, and accumulation of $\mathrm{OH}^{-}$ within the well would block water permeation. It is thus of particular interest to investigate how the aquaporin prevents $\mathrm{OH}^{-}$or other negatively charged ions from being attracted to the NPA region of the pore. Indeed, when placed near the center of the pore, hydroxide ions are indeed attracted towards the NPA region of the pore (Figure 4, bottom). When placed farther away from the central part of the pore, however, $\mathrm{OH}^{-}$ are no longer attracted by the NPA region but, instead, are expelled from the pore.

Figure 5 also suggests that the mechanism of hydroxide exclusion is mainly electrostatic in nature, caused by two barriers on both sides of the NPA region (near $30 \AA$ and $70 \AA$, respectively), which appear as local minima in the proton free energy and electrostatic profile (Figure 6(a)). Note, however, that the free energy profile for hydroxide ions is not necessarily the negated proton free energy profile, as the mechanisms for proton and hydroxide conduction are very different. ${ }^{46}$ However, if the $\mathrm{OH}^{-}$barrier is dominated by electrostatics as is the proton barrier, then the (negated) proton free energy profile can be interpreted as an approximate free energy profile for hydroxide ions. In this case, one would estimate an $\mathrm{OH}^{-}$barrier height of approximately $10 \mathrm{~kJ} \mathrm{~mol}^{-1}$, which at first sight seems too low to prevent hydroxide ions from getting trapped in the NPA region of the pore. Note, however, that there is an entropic cost connected to moving from bulk into the narrow channel, just like for protons, as described above, which leads to the observed exclusion of $\mathrm{OH}^{-}$from the pore (Figure 4, bottom).

\section{Control calculations}

We have carried out a number of additional calculations. First, to be able to compare the purely diffusive motion of $\mathrm{H}_{3} \mathrm{O}^{+}$and $\mathrm{OH}^{-}$, as described by conventional MD simulations with the Q-HOP MD simulations. Second, to be able to estimate the amount of statistical error in each of the presented energy profiles as well as their reproducibility.

As can be seen from the non-equilibrium trajectories (Figures 2 and 4) as well as from the distributions of protons, $\mathrm{H}_{3} \mathrm{O}^{+}$, and $\mathrm{OH}^{-}$positions along the pore (Figure 5), there is good qualitative agreement between the Q-HOP and conventional MD simulations, albeit that the proton kinetics is much faster in the Q-HOP simulations due to the ability of protons to be involved in proton transfer reactions. Because of this, the Q-HOP simulations (and the derived proton free energy profile) are affected not only by the classical free energy determinants (predominantly electrostatics and entropic effects), but also by additional factors that specifically affect proton transfer, most notably the intactness of a hydrogen-bonded chain. Indeed, although the proton free energy profile is dominated by electrostatic effects (Figure 6(a)), the secondary proton barrier in the ar/R constriction region is found to be caused by a frequent interruption of the hydrogen-bonded chain (Figure 6(b)).

To further decompose the proton free energy profile into individual components, the next step is to estimate the electrostatic contribution. The determination of the electrostatic potential that protons (in the form of $\mathrm{H}_{3} \mathrm{O}^{+}$) experience on their way across the pore, however, is complicated by the fact that the electrostatic component of the force (Figure $7(\mathrm{~b})$, red curve) is, on average, an order of magnitude larger than the total net force that these ions experience (blue curve and green circles). Closer inspection showed that this effect is caused mainly by strong short-range electrostatic interactions (typically hydrogen bonds) that are compensated largely by repulsive Pauli interactions. Therefore, the net force on an ion in the pore is typically much smaller than the electrostatic component of the force, even in the case when the underlying potential was mainly electrostatic in nature. This situation makes it problematic to calculate the effective electrostatic potential profile across the pore. However, one may assume that this short-range compensation varies only slightly in strength along the pore (as will be analyzed and justified further below). In this case, the net force acting on an $\left(\mathrm{H}_{3} \mathrm{O}^{+}\right)$ion will indeed allow us to estimate the effective electrostatic potential felt by the proton.

This mean force acting on $\mathrm{H}_{3} \mathrm{O}^{+}$was determined in a number of ways. The method of choice would be to calculate a true PMF over a sufficient number of constrained or umbrella positions of the ion in the pore. Because of the observed slow convergence, however, we used the calculations using probe charges at suitable water positions instead, which requires a careful estimate of the error associated with it (see also Methods). The obvious way to do this is to select a subset for comparison with the computationally expensive method of choice. To this end, umbrella sampling simulations were started in which $\mathrm{H}_{3} \mathrm{O}^{+}$were kept fixed with a harmonic potential at 20 different positions along the pore (see Methods). As can be seen in Figure $7(\mathrm{~b})$, the mean umbrella forces correlate well with the mean electrostatic force profile. Note that despite simulation times of up to $5 \mathrm{~ns}$ for each single umbrella sampling simulation, these forces scatter considerably, which underscores the indeed slow convergence that made the direct approach untractable. The slow convergence is mainly due to the slow equilibration of neighboring water molecules and protein side-chains in the pore. 


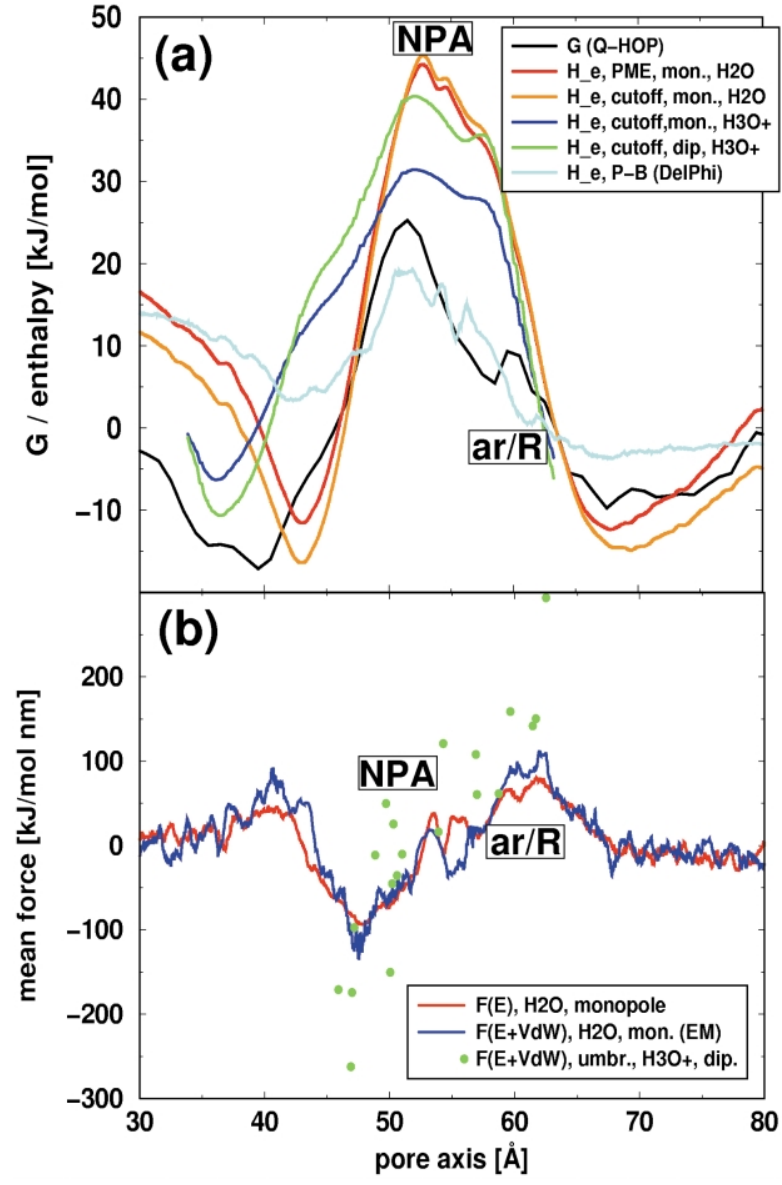

Figure 7. Control calculations. (a) The maximum likelihood proton free energy profile (black) together with the electrostatic potential profiles obtained with the different methods described in the text. The red and orange curves show the electrostatic potential calculated from the mean force on a positive unit charge, determined at different water positions along the pore, using PME and a cut-off of $1.2 \mathrm{~nm}$, respectively. Electrostatic profiles calculated from the mean force at actual $\mathrm{H}_{3} \mathrm{O}^{+}$positions taken from Q-HOP simulations are shown in blue and green, respectively. The blue curve was determined, like the red and orange curves, with a positive probe unit charge on the oxygen position, whereas the green curve is based on the actual dipole charges of the $\mathrm{H}_{3} \mathrm{O}^{+}$. The cyan curve shows a continuum Poisson-Boltzmann electrostatic profile calculated with DelPhi, ${ }^{47}$ averaged over multiple MD snapshots, using water oxygen positions from passing water molecules as probe positions. (b) Mean force profiles as a function of the pore axis. Shown (in red) is the mean electrostatic force on a positive probe unit charge on the oxygen position of water molecules that passed the pore in an equilibrium MD simulation (integration of this curve yields the red curve in (a)), and (in blue) the total non-bonded (electrostatic and Lennard-Jones) force (after energyminimization) at the same positions. The red curve was scaled (by 0.127 ) to fit the blue curve. The green points depict mean forces on $\mathrm{H}_{3} \mathrm{O}^{+}$in the pore direction obtained from multi-nanosecond umbrella sampling simulations during which the oxygen atom of the $\mathrm{H}_{3} \mathrm{O}^{+}$ was fixed by an umbrella potential along the $z$ direction at 20 different positions along the pore.
In full agreement with the forces obtained for the probe point charge (Figure $7(\mathrm{~b})$ ) the electrostatic component of the force acting on the $\mathrm{H}_{3} \mathrm{O}^{+}$in the umbrella simulations is larger by a factor of about 12 than the total force acting on the $\mathrm{H}_{3} \mathrm{O}^{+}$, as obtained from a least-squares fit between the two quantities. A similar ratio was obtained when the force profile from the water positions was recalculated not only including the electrostatic, but also the Lennard-Jones components (Figure 7(b)), after energy minimization. All electrostatics profiles that were solely calculated based on the electrostatic component of the mean force were therefore scaled (see also Methods).

As discussed above, the electrostatics profile as determined by integrating the mean force on a probe charge on water positions is generally expected to deviate from a PMF. Although for AQP1 the above control calculations suggest that these deviations are relatively small, a number of specific potential sources of deviation deserve particular attention: (a) the fact that water positions instead of true proton positions are taken as probe positions; (b) the approximation of the $\mathrm{H}_{3} \mathrm{O}^{+}$ charge by a point charge (whereas $\mathrm{H}_{3} \mathrm{O}^{+}$is a dipole); and (c) the lack of relaxation (most notably the alignment of neighboring dipoles) of the surroundings due to the presence of a positive charge in the pore.

To check to what extent the calculated profile is affected by the chosen set of positions as well as by the pore environment, we have compared the profile obtained with water positions with a profile obtained from snapshots of the Q-HOP simulations, i.e. including true $\mathrm{H}_{3} \mathrm{O}^{+}$positions (Figure 7). Although the statistics for the latter is worse than for the former (493 versus 17,255 positions, respectively), the profiles are qualitatively similar, especially near the main NPA barrier. The only significant deviations are seen near the first minimum at $40 \AA$. Interestingly, this minimum is shifted by about $6 \AA$ to the intracellular side with respect to the profile calculated from the water positions, and now better agrees with the minimum in the free energy profile obtained from the Q-HOP simulations. This suggests that the paths taken by protons and water molecules are relatively similar to each other, except for the ar/ $R$ constriction region, where the proton was found to hop across His182 in 15 of the 20 cases where the proton left the pore on the extracellular side. Moreover, it shows that the electrostatic field inside the pore, that causes water molecules to strongly align inside the pore, is caused mainly by the protein, and is perturbed only weakly by an excess positive charge. Additionally, this result shows that the neglect of a more physiological protein environment (tetramer, membrane), as well as the applied position restraints on $\mathrm{C}^{\alpha}$ atoms in the Q-HOP simulations does not cause significant artefacts. This is also true for the use of a cut-off (12 $\AA$ radius) in the Q-HOP simulations, which, as shown in in Figure 7(a), yields energy profiles that 
are similar to those calculated with full electrostatics (particle-mesh Ewald, PME).

We note that the Q-HOP method in its current implementation cannot explicitly account for concerted proton hops, and currently only hops to water and to histidine are implemented. Although we do not expect these limitations to significantly affect our results (see also Methods), we note that if these features were implemented, this would lead to an enhancement of the proton transfer rates, and a corresponding lowering of the hydrogen bond-mediated proton transfer barriers, rendering the role of electrostatics even more pronounced.

We compared the electrostatic energy of a monopole (like the probe charge used to obtain the profile) to the energy of a dipole (like $\mathrm{H}_{3} \mathrm{O}^{+}$) by calculating the mean force on $\mathrm{H}_{3} \mathrm{O}^{+}$positions in Q-HOP simulations both as a dipole (Figure 7(a), blue curve) and as a monopole (like for probe charges at water positions, Figure 7(a), green curve). The fact that the two profiles are rather similar indicates that the monopole dominates the electrostatic profile for a $\mathrm{H}_{3} \mathrm{O}^{+}$.

An independent estimate for the electrostatic potential in the pore was calculated using a continuum Poisson-Boltzmann approach, as implemented in the DelPhi $\operatorname{program}^{47}$ (see Methods). The potential was calculated by averaging over a large number of MD snapshots of the AQP1 tetrameric structure, by evaluating the potential at the same water oxygen positions that were used above for calculating the other electrostatic potential profiles. The agreement with the other electrostatic profiles (Figures 6(a) and 7(a)) underscores the role of electrostatics for the mechanism of proton exclusion.

\section{Conclusions}

Our results from a combination of Q-HOP simulations, which explicitly describe proton transfer reactions, and multinanosecond conventional molecular dynamics simulations, strongly indicate that proton exclusion from the aquaporin pores is predominantly achieved by a strong electrostatic barrier. The main barrier of approximately 25$30 \mathrm{~kJ} \mathrm{~mol}^{-1}$ is predicted to be located at the center of the pore, near the NPA fingerprint region. Here, the positive $\mathrm{N}$-terminal ends of the macro-dipoles of helices B and E meet, which are the main determinants of the electrostatic barrier. Interruption of the hydrogen-bonded chain of water molecules through the pore causes a secondary barrier located at the ar/ $\mathrm{R}$ constriction region. The calculated barrier height for protons is approximately as high as in typical lipid bilayers, and suffices to prevent leakage of the electrochemical gradient across the membrane. The aquaporin channel not only efficiently blocks protons and hydronium ions, but also negatively charged hydroxide ions. This is proposed to be achieved by a combination of electrostatic barriers and entropic effects, which is essential not only for preventing the dissipation of the electrochenical gradient, but also for the function of water channel because it avoids hydroxide ions from being trapped within the positively charged NPA region, which would otherwise block the channel.

\section{Methods}

\section{Conventional MD simulations}

Conventional MD simulations with excess $\mathrm{H}_{3} \mathrm{O}^{+}$and $\mathrm{OH}^{-}$were carried out with the gromacs $\dagger^{48}$ simulation software. The simulation set-up and conditions were similar to those described before. ${ }^{35}$ As a starting conformation, the bovine aquaporin-1 (bAQP1) X-ray structure $^{28}$ (Figure 1(a)) was placed as a tetramer centrally into a palmitoyloleoylphosphatidylethanolamine (POPE) lipid bilayer patch of 272 lipids, and was solvated on both sides with 19,442 SPC water molecules ${ }^{49}$ in total (cf. Figure 1(b)). The gromos $87^{50}$ force-field with modifications ${ }^{51}$ and explicit polar and aromatic hydrogen atoms was used. Lipid parameters were taken from Berger et al.: $:^{52} 12$ chloride ions were added to the periodic simulation box to neutralize the net positive charge of the protein, rendering the total system size 81,814 atoms. After energy minimization, a short simulation with position restraints of $1000 \mathrm{~kJ} \mathrm{~mol}^{-1} \mathrm{~nm}^{-2}$ on the non-hydrogen protein atoms was carried out to relax the lipids and water molecules around the protein. Subsequently, the system was allowed to equilibrate for $2 \mathrm{~ns}$ before $\mathrm{H}_{3} \mathrm{O}^{+}$and $\mathrm{OH}^{-}$were added. Additionally, the simulation without excess $\mathrm{H}_{3} \mathrm{O}^{+}$and $\mathrm{OH}^{-}$was extended to a total time of $10 \mathrm{~ns}$. During these simulations, the temperature was kept constant by weakly ( $\tau=0.1 \mathrm{ps})$ coupling the protein, lipids, and solvent separately to a temperature bath ${ }^{53}$ of $300 \mathrm{~K}$. Likewise, the pressure was kept constant by weakly coupling the system to a pressure bath of 1 bar. The $x y$ (membrane plane) and $z$ (membrane normal) directions, respectively, were separately coupled with a coupling constant $\tau$ of 1 ps. Electrostatic interactions were calculated with the PME method. ${ }^{54}$ Pauli and van der Waals interactions were described with a Lennard-Jones potential, which was cut off at $1.2 \mathrm{~nm}$. The Settle ${ }^{55}$ algorithm was used to constrain the bond lengths and angles of the water molecules, and lincs ${ }^{56}$ was used to constrain all other bond lengths, allowing a time-step of $2 \mathrm{fs}$. Four simulations with four $\mathrm{H}_{3} \mathrm{O}^{+}$each (one per pore), as well as two simulations with four and eight $\mathrm{OH}^{-}$, respectively, were carried out. Each of these simulations had a length of $1.5 \mathrm{~ns}$. The charges of $\mathrm{H}_{3} \mathrm{O}^{+}$were taken from earlier work, ${ }^{37}$ obtained by a restrained ESP-fit using NWChem. The oxygen charge is -0.749 and the hydrogen charges are +0.583 . Likewise, the charges of $\mathrm{OH}^{-}$were taken from earlier work, ${ }^{57}$ where their magnitude was adapted according to the average electrostatic potential field felt in the protein to match the quantum mechanical dipole moment of an $\mathrm{OH}^{-}$ion in an electrostatic field field of equal size. ${ }^{58}$ The oxygen charge is -1.3 and the proton carries a charge of +0.3 .

$\dagger$ http:/ / www.gromacs.org 


\section{Q-HOP MD simulations}

To explicitly simulate quantum mechanical and thermally activated proton transfer reactions between water molecules inside the pore, Q-HOP simulations ${ }^{37}$ were carried out with a modified version of the ARGOS simulation package. ${ }^{59}$ For the Q-HOP simulations, the bAQP1 X-ray structure ${ }^{28}$ was solvated in a rectangular periodic simulation box containing 6303 SPC water molecules; ${ }^{49}$ this simulation system comprised a total of 22,678 atoms (Figure 1(c)). After energy minimization, an equilibration simulation of 250 ps was carried out, from which snapshots were collected every 20 ps. From each of these snapshots, four different water molecules in the pore region were selected for protonation. Each of the obtained 48 configurations was energy minimized and then equilibrated for 1 ps. During that period, the oxygen atom of the protonated $\mathrm{H}_{3} \mathrm{O}^{+}$was kept fixed using a harmonic potential with a force constant of $1000 \mathrm{~kJ} \mathrm{~mol}^{-1} \mathrm{~nm}^{-2}$. From each of the obtained configurations, a Q-HOP simulation with a length of 100 ps was carried out. To prevent structural rearrangements due to the non-physiological environment of the protein (i.e. a monomer solvated in water instead of a tetramer embedded in a lipid bilayer), all $\mathrm{C}^{\alpha}$ positions of the protein were position-restrained with a force constant of $1000 \mathrm{~kJ} \mathrm{~mol}^{-1} \mathrm{~nm}^{-2}$. In all ARGOS simulations, the Amber95 force-field ${ }^{60}$ was used. All non-bonded interactions (electrostatic and Lennard-Jones) were cut off at $1.2 \mathrm{~nm}$. Temperature and pressure were kept constant by coupling to an external bath ${ }^{53}$ of $300 \mathrm{~K}$ and $1 \mathrm{bar}$ with coupling constants of 0.4 and $0.5 \mathrm{ps}$, respectively. The $\mathrm{SHAKE}^{61}$ algorithm was used to constrain bond lengths, allowing a time-step of $2 \mathrm{fs}$.

The Q-HOP molecular dynamics procedure was employed as described. ${ }^{37,62-64}$ In this method, stochastic proton hopping events are included in otherwise standard molecular dynamics simulations. Previous applications successfully addressed the diffusion of an excess proton in water, ${ }^{37}$ the protonation equilibrium of Asp in a solvent box, ${ }^{37}$ and the three-step proton relay in green fluorescent protein. ${ }^{57}$ Energy barriers for proton transfer were carefully parameterized against quantum chemical calculations on donor-acceptor model systems as simple functions of the donor-acceptor distance and of the relative energy difference between the donorbound and the acceptor-bound states. ${ }^{64}$ Transition state theory is then employed to compute transfer probabilities over large barriers, and pre-calculated transmission coefficients from wave-packet dynamics calculations are applied for the transfer over small barriers. ${ }^{63}$ We want to stress that transfers in the regime of larger energy barriers are very unlikely during typical MD simulations of 100 ps length. In this work, transfers were only observed at donor-acceptor distances closer than $2.7 \AA$. The efficiency of the method results from the fact that the parameterizations have been precomputed and can almost instantaneously be evaluated during the MD simulation for any given donor-acceptor configuration, properly taking into account electrostatic stabilization by the environment. Proton hopping is allowed every $10 \mathrm{fs}$, which is the approximate time required for a quantum wave-packet to cross a small energy barrier. Although concerted proton transfer is not currently implemented in the Q-HOP algorithm, the diffusion rate of an excess proton in bulk water could be well reproduced. ${ }^{37}$ In this work, proton transfer is only allowed between water molecules as well as to the amino acid histidine. Although it would be technically possible to include transfers to other protein residues once the corresponding parameterizations are derived (work in progress), for the present study, proton hops to and from amino acid residues other than histidine are not implemented. Note that both approximations may lead to a slight overestimation of the proton transfer free energy barriers derived from Q-HOP MD. Coordinates of the protonated water molecule were recorded every MD step for subsequent analysis.

\section{Electrostatic potential calculations}

The electrostatic potential along the pore axis was estimated using three different approaches. First, the mean electrostatic force on a probe charge at different positions of water molecules inside the pore during MD simulations was calculated. Second, the electrostatic and total mean force on $\mathrm{H}_{3} \mathrm{O}^{+}$at different positions in the pore during umbrella sampling simulations was determined. Finally, the electrostatic potential was estimated by a continuum Poisson-Boltzmann approach. ${ }^{47}$

\section{The average electrostatic field at water positions}

The electrostatic force on a positive probe unit charge was evaluated at the positions of water molecules passing the pore during the $10 \mathrm{~ns}$ equilibrium MD simulation of the bAQP1 tetramer embedded in a solvated POPE bilayer described above. In total, 17,255 water molecule positions from $1000 \mathrm{MD}$ snapshots were selected such that a homogeneous distribution of positions along the pore axis was obtained. For each of the positions, the selected water molecule was replaced by a positive probe unit charge located at the dipole center of the water molecule (i.e. the midpoint between the oxygen atom and the midpoint between the two hydrogen atoms). For each of the obtained configurations (with an excess positive probe charge) the electrostatic component of the force on the probe charge was evaluated. For comparison, the long-range contribution of this force was calculated both with a cut-off approach (using a cut-off radius of $1.2 \mathrm{~nm}$ ) and using the PME method. In the case of the PME calculation, in addition to the excess positive probe charge, a complementary negative charge was added in the bulk water region, to ensure electrostatic neutrality. The mean component of the force in the pore direction (z-axis) was evaluated as a function of the probe axis, using a Gaussian filter with a width of $0.1 \mathrm{~nm}$. This mean force was then integrated to obtain an estimated electrostatic potential profile. Note that this averaged potential does not include the reaction field that would actually be caused by a real charge, and therefore is expected to overestimate the true electrostatic potential. Yet, assuming that the polarization effect of the surrounding is of similar size along the channel, this estimate will provide a qualitative picture. To capture the main part of the reaction field, a more expensive calculation of the electrostatic profile was also carried out. Here, rearrangements of the surroundings as a response to the introduced particle were considered by energy-minimization of each of the configurations using a steepest descent algorithm (100 steps each) prior to the force evaluation. This required including also Lennard-Jones interactions with the probe charge, using the Lennard-Jones parameters of a water oxygen atom. A least-squares fit of the profiles with and without the Lennard-Jones contribution shows that the shapes of the force profiles are very similar (Figure 7) but, as was 
expected, the (inexpensive) electrostatics-only profile overestimates the electrostatic contribution by a factor of approximately 7.9. Therefore, all force profiles in which only the electrostatic component of the nonbonded force was evaluated were subsequently scaled with this factor.

A further correction of the obtained electrostatic potential profile was necessary because the inserted probe charges were described as monopoles, whereas the particle of interest, $\mathrm{H}_{3} \mathrm{O}^{+}$, has a dipole. Moreover, the selected probe positions were positions of water molecules that spontaneously passed the pore in an equilibrium MD simulation. It is conceivable that a passing $\mathrm{H}_{3} \mathrm{O}^{+}$or proton would take a different path, on average, than a water molecule. To estimate the size of these two effects, the electrostatic potential was also calculated for $\mathrm{H}_{3} \mathrm{O}^{+}$positions in snapshots from Q-HOP simulations, both treated as monopoles and dipoles (see Figure 7).

\section{Umbrella sampling simulations}

Note that the methods described above do not yield a potential of mean force (PMF) in the strict statistical sense, mainly because it lacks entropic contributions. To estimate their size, we additionally evaluated the statistically correct PMF acting on a $\mathrm{H}_{3} \mathrm{O}^{+}$along the channel axis by five umbrella sampling calculations, each with four excess $\mathrm{H}_{3} \mathrm{O}^{+}$(one per pore). In these calculations, the $z$-coordinate of the oxygen atom of the respective $\mathrm{H}_{3} \mathrm{O}^{+}$was restrained using a harmonic potential with a force constant of $10,000 \mathrm{~kJ} \mathrm{~mol}^{-1} \mathrm{~nm}^{-2}$. In addition, the center of mass of each of the monomeric bAQP1 channels was kept fixed. We used a stiff restraint instead of a applying a constraint force, since the combination of a one-dimensional external constraint in addition to the internal bond constraints is not currently implemented in the gromacs software.

Since these calculations do not suffer from the same drawbacks as the calculation of the mean electrostatic force acting on a probe charge as described above (predominantly entropic effects and the relaxation of neighboring dipoles), they would be the method of choice to calculate a true PMF by integrating over many positions along the pore. The problem with this approach in the present case, however, was an unusually slow convergence, requiring multi-nanosecond simulations to obtain sufficiently accurate results. As the main reason for such slow convergence we identified slow reorientations of the $\mathrm{H}_{3} \mathrm{O}^{+}$dipole and a slow response of the surroundings, particularly due to protein side-chain reorientations and the presence and orientation of neighboring water molecules. Therefore, we could only determine the mean force at a relatively small number of positions (see Figure 7) rather than along the complete pore. However, these data sufficed to allow comparison with the purely electrostatic profiles as well as with the non-equilibrium free energy profile estimate described further below.

\section{Continuum Poisson-Boltzmann calculations}

A continuum Poisson-Boltzmann electrostatic potential profile was also calculated with the DelPhi program. ${ }^{47}$ Again, protein conformations collected (every $10 \mathrm{ps}$ ) from the equilibrium $10 \mathrm{~ns}$ MD simulation were taken as input structures. The oxygen positions of passing water molecules were taken as probe positions, as in the calculation of the force profiles described above. The profile shown in Figures 6(a) and 7(a) was obtained by averaging over 1000 snapshot profiles. The dielectric constants were chosen to be 80 and 4 , for protein exterior and interior, respectively.

The molecular graphics in Figures 1-3 were created with bobscript ${ }^{65,66}$ and Raster3D. ${ }^{67}$

\section{Acknowledgements}

B.L.dG. was supported by the BIOTECH program of the EU, grants QLRT 2000/00778 and 2000/00504. We thank Tjerk Straatsma for permission to use the ARGOS program, and Markus Lill for technical support with ARGOS.

\section{References}

1. Mitchell, P. (1961). Coupling of phosphorylation to electron and hydrogen transfer by a chemi-osmotic type of mechanism. Nature, 191, 144-148.

2. Preston, G. M., Carroll, T. P., Guggino, W. B. \& Agre, P. (1992). Appearance of water channels in Xenopus oocytes expressing red-cell CHIP28 protein. Science, 256, 385-387.

3. Zeidel, M. L., Ambudkar, S. V., Smith, B. L. \& Agre, P. (1992). Reconstitution of functional water channels in liposomes containing purified red-cell CHIP28 protein. Biochemistry, 31, 7436-7440.

4. Zeidel, M. L., Nielsen, S., Smith, B. L., Ambudkar, S. V., Maunsbach, A. B. \& Agre, P. (1994). Ultrastructure, pharmacological inhibition, and transport selectivity of aquaporin channel-forming integral protein in proteoliposomes. Biochemistry, 33, $1606-1615$.

5. Agre, P., Bonhivers, M. \& Borgnia, M. J. (1998). The aquaporins, blueprints for cellular plumbing systems. J. Biol. Chem. 273, 14659-14662.

6. Borgnia, M., Nielsen, S., Engel, A. \& Agre, P. (1999). Cellular and molecular biology of the aquaporin water channels. Annu. Rev. Biochem. 68, 425-458.

7. Fujiyoshi, Y., Mitsuoka, K., de Groot, B. L., Philippsen, A., Grubmüller, H., Agre, P. \& Enge, A. (2002). Structure and function of water channels. Curr. Opin. Struct. Biol. 12, 509-515.

8. Pomès, R. \& Roux, B. (1996). Structure and dynamics of a proton wire: a theoretical study of $\mathrm{H}^{+}$translocation along the single-file water chain in the gramicidin A channel. Biophys. J. 71, 19-39.

9. Pomès, R. \& Roux, B. (1998). Free energy profiles for $\mathrm{H}^{+}$conduction along hydrogen-bonded chains of water molecules. Biophys. J. 75, 33-40.

10. Marx, D., Tuckerman, M. E., Hutter, J. \& Parrinello, M. (1999). The nature of the hydrated excess proton in water. Nature, 397, 601-604.

11. Pomès, R. \& Roux, B. (2002). Molecular mechanism of $\mathrm{H}^{+}$conduction in the single-file water chain of the gramicidin channel. Biophys. J. 82, 2304-2316.

12. de Grotthuss, C. J. T. (1806). Sur la dècomposition de l'eau et des corps qu'elle tient en dissolution à l'aide de l'èlectricitè galvanique. Ann. Chim. 58, 54-74.

13. Agmon, N. (1995). The Grotthuss mechanism. Chem. Phys. Letters, 244, 456-462.

14. Tuckerman, M. E., Marx, D., Klein, M. \& Parrinello, M. (1997). On the quantum nature of the shared proton in hydrogen bonds. Science, 275, 817-820. 
15. Akeson, M. \& Deamer, D. W. (1991). Proton conductance by the gramicidin water wire. Biophys. J. 60, 101-109.

16. Dellago, C., Naor, M. M. \& Hummer, G. (2003). Proton transport through water-filled carbon nanotubes. Phys. Rev. Letters, 90, 105902/1-105902/4.

17. Kandori, H., Yamazaki, Y., Sasaki, J., Needleman, R., Lanyi, J. K. \& Maeda, A. (1995). Water-mediated proton-transfer in proteins-an FTIR study of bacteriorhodopsin. J. Am. Chem. Soc. 117, 2118-2119.

18. Luecke, H., Schobert, B., Richter, H. T., Cartailler, J. P. \& Lanyi, J. K. (1999). Structure of bacteriorhodopsin at 1.55 Angstrom resolution. J. Mol. Biol. 291, 899-911.

19. Belrhali, H., Nollert, P., Royant, A., Menzel, C., Rosenbusch, J., Landau, E. M. \& Pebay-Peyroula, E. (1999). Protein, lipid and water organization in bacteriorhodopsin crystals: a molecular view of the purple membrane at 1.9 Ångstrom resolution. Structure, 7, 909-917.

20. Baudry, J., Tajkhorshid, E., Molnar, F., Phillips, J. \& Schulten, K. (2001). Molecular dynamics study of bacteriorhodopsin and the purple membrane. J. Phys. Chem. B, 105, 905-918.

21. Deamer, D. W. \& Nichols, J. W. (1989). Proton flux mechanisms in model and biological membranes. J. Membr. Biol. 107, 91-103.

22. Marrink, S.-J., Jähnig, F. \& Berendsen, H. J. C. (1996). Proton transport across transient single-file water pores in a lipid a lipid membrane studied by molecular dynamics simulations. Biophys. J. 71, 632-647.

23. Deen, P. M. T. \& van Os, C. H. (1998). Epithelial aquaporins. Curr. Opin. Cell. Biol. 10, 435-442.

24. Li, J. \& Verkman, A. S. (2001). Impaired hearing in mice lacking aquaporin-4 water channels. J. Biol. Chem. 276, 31233-31237.

25. Heller, K. B., Lin, E. C. \& Wilson, T. H. (1980). Substrate-specificity and transport-properties of the glycerol facilitator of Escherichia coli. J. Bacteriol. 144, 274-278.

26. Murata, K., Mitsuoka, K., Walz, T., Agre, P., Heymann, J., Engel, A. \& Fujiyoshi, Y. (2000). Structural determinants of water permeation through Aquaporin-1. Nature, 407, 599-605.

27. de Groot, B. L., Engel, A. \& Grubmüller, H. (2001). A refined structure of human Aquaporin-1. FEBS Letters, 504, 206-211.

28. Sui, H., Han, B.-G., Lee, J. K., Walian, P. \& Jap, B. K. (2001). Structural basis of water-specific transport through the AQP1 water channel. Nature, 414, 872-878.

29. Fu, D., Libson, A., Miercke, L. J., Weitzman, C., Nollert, P., Krucinski, J. \& Stroud, R. M. (2000). Structure of a glycerol-conducting channel and the basis for its selectivity. Science, 290, 481-486.

30. Verbavatz, J. M., Brown, D., Sabolic, I., Valenti, G., Siello, D. A., Van Hoek, A. N., Ma, T. \& Verkman, A. S. (1993). Tetrameric assembly of CHIP28 water channels in liposomes and cell-membranes-a freeze-fracture study. J. Cell Biol. 123, 605-618.

31. Shi, L. B., Skach, W. R. \& Verkman, A. S. (1994). Functional independence of monomeric CHIP28 water channels revealed by expression of wild-typemutant heterodimers. J. Biol. Chem. 269, 10417-10422.

32. Jung, J. S., Preston, G. M., Smith, B. L., Guggino, W. B. \& Agre, P. (1994). Molecular structure of the water channel through Aquaporin CHIP-the hourglass model. J. Biol. Chem. 269, 14648-14654.
33. Mitsuoka, K., Murata, K., Walz, T., Hirai, T., Agre, P., Heymann, J. B. et al. (1999). The structure of Aquaporin- 1 at $4.5 \AA$ resolution reveals short $\alpha$-helices in the center of the monomer. J. Struct. Biol. 128, 34-43.

34. Hol, W. G. J., van Duijnen, P. T. \& Berendsen, H. J. C. (1978). The $\alpha$-helix dipole and the properties of proteins. Nature, 273, 443-446.

35. de Groot, B. L. \& Grubmüller, H. (2001). Water permeation across biological membranes: mechanism and dynamics of Aquaporin-1 and GlpF. Science, 294, 2353-2357.

36. Tajkhorshid, E., Nollert, P., Jensen, M.Ø., Miercke, L. J. W., O'Connell, J., Stroud, R. M. \& Schulten, K. (2002). Control of the selectivity of the aquaporin water channel family by global orientational tuning. Science, 296, 525-530.

37. Lill, M. A. \& Helms, V. (2001). Molecular dynamics simulation of proton transport with quantum mechanically derived proton hopping rates (Q-HOP MD). J. Chem. Phys. 115, 7993-8005.

38. Smondyrev, A. M. \& Voth, G. A. (2002). Molecular dynamics simulation of proton transport through the influenza A virus M2 channel. Biophys. J. 83, 1987-1996.

39. Nichols, J. W., Hill, M. W., Bangham, A. D. \& Deamer, D. W. (1980). Measurement of net protonhydroxyl permeability of large unilamellar liposomes with the fluorescent $\mathrm{pH}$ probe, 9-aminoacridine. Biochim. Biophys. Acta, 596, 393-403.

40. Deamer, D. W. \& Nichols, J. W. (1983). Proton hydroxide permeability of liposomes. Proc. Natl Acad. Sci. USA, 80, 165-168.

41. Gutknecht, J. (1987). Proton hydroxide conductance and permeability through phospholipid-bilayer membranes. Proc. Natl Acad. Sci. USA, 84, 6443-6446.

42. Paula, S., Volkov, A. G., Hoek, A. N. V., Haines, T. H. \& Deamer, D. W. (1996). Permeation of protons, potassium ions, and small polar molecules through phospholipid bilayers as a function of membrane thickness. Biophys. J. 70, 339-348.

43. Carruthers, A. \& Melchior, D. L. (1983). Studies of the relationship between bilayer water permeability and bilayer physical state. Biochemistry, 22, 5797-5807.

44. Jansen, M. \& Blume, A. (1995). A comparative study of diffusive and osmotic water permeation across bilayers composed of phospholipids with different head groups and fatty acyl chains. Biophys. J. 68, 997-1008.

45. Marrink, S.-J. \& Berendsen, H. J. C. (1994). Simulation of water transport through a lipid-membrane. J. Phys. Chem. 98, 4155-4168.

46. Tuckerman, M. E., Marx, D. \& Parrinello, M. (2002). The nature and transport mechanism of hydrated hydroxide ions in aqueous solution. Nature, 417, 925-929.

47. Klapper, I., Hagstrom, R., Fine, R., Sharp, K. \& Honig, B. (1986). Focusing of electric fields in the active site of $\mathrm{Cu}-\mathrm{Zn}$ superoxide dismutase: effects of ionic strength and amino-acid modification. Proteins: Struct. Funct. Genet. 1, 47-59.

48. Lindahl, E., Hess, B. \& Van der Spoel, D. (2001). GROMACS 3.0: a package for molecular simulation and trajectory analysis. J. Mol. Model. 7, 306-317.

49. Berendsen, H. J. C., Postma, J. P. M., van Gunsteren, W. F. \& Hermans, J. (1981). Interaction models for water in relation to protein hydration. In 
Intermolecular Forces (Pullman, B., ed.), pp. 331-342, Reidel Publishing Company, Dordrecht.

50. Van Gunsteren, W. F. \& Berendsen, H. J. C. (1987) Gromos Manual, BIOMOS, Biomolecular Software, Laboratory of Physical Chemistry, University of Groningen, The Netherlands.

51. Van Buuren, A. R., Marrink, S. J. \& Berendsen, H. J. C. (1993). A molecular dynamics study of the decane/water interface. J. Phys. Chem. 97, 9206-9212.

52. Berger, O., Edholm, O. \& Jähnig, F. (1997). Molecular dynamics simulations of a fluid bilayer of dipalmitoylphosphatidylcholine at full hydration, constant pressure, and constant temperature. Biophys. J. 72, 2002-2013.

53. Berendsen, H. J. C., Postma, J. P. M., DiNola, A. \& Haak, J. R. (1984). Molecular dynamics with coupling to an external bath. J. Chem. Phys. 81, 3684-3690.

54. Darden, T., York, D. \& Pedersen, L. (1993). Particle mesh Ewald: an $N \log (N)$ method for Ewald sums in large systems. J. Chem. Phys. 98, 10089-10092.

55. Miyamoto, S. \& Kollman, P. A. (1992). SETTLE: an analytical version of the SHAKE and RATTLE algorithms for rigid water models. J. Comput. Chem. 13, 952-962.

56. Hess, B., Bekker, H., Berendsen, H. J. C. \& Fraaije, J. G. E. M. (1997). LINCS: a linear constraint solver for molecular simulations. J. Comput. Chem. 18, 1463-1472.

57. Lill, M. A. \& Helms, V. (2002). Proton shuttle in green fluorescent protein studied by dynamic simulations. Proc. Natl Acad. Sci. USA, 99, 2778-2781.

58. Hermansson, K. (1995). O-H bonds in electricfields-electron-densities and vibrational frequencyshifts. Chem. Phys. Letters, 233, 376-382.

59. Straatsma, T. P. \& McCammon, J. A. (1990). ARGOS, a vectorized general molecular dynamics program. J. Comput. Chem. 11, 943-951.

60. Cornell, W. D., Cieplak, P., Bayly, C. I., Gould, I. R., Merz, K. M., Ferguson, D. M. et al. (1995). A 2nd generation force-field for the simulation of proteins, nucleic-acids, and organic-molecules. J. Am. Chem. Soc. 117, 5179-5197.

61. Ryckaert, J. P., Ciccotti, G. \& Berendsen, H. J. C (1977). Numerical integration of the cartesian equations of motion of a system with constraints; molecular dynamics of $n$-alkanes. J. Comput. Phys. 23, 327-341.

62. Lill, M. A., Hutter, M. C. \& Helms, V. (2000). Accounting for environmental effects in $a b$ initio calculations of proton transfer barriers. J. Phys. Chem. A, 104, 8283-8289.

63. Lill, M. A. \& Helms, V. (2001). Reaction rates for proton transfer over small barriers and connection to transition state theory. J. Chem. Phys. 115, 7985-7992.

64. Lill, M. A. \& Helms, V. (2001). Compact parameter set for fast estimation of proton transfer rates. J. Chem. Phys. 114, 1125-1132.

65. Kraulis, P. J. (1991). MOLSCRIPT: a program to produce both detailed and schematic plots of protein structures. J. Appl. Crystallog. 24, 946-950.

66. Esnouf, R. M. (1997). An extensively modified version of molscript that includes greatly enhanced coloring capabilities. J. Mol. Graph. Model. 15, 132-134

67. Merritt, E. A. \& Bacon, D. J. (1997). Raster3D: photorealistic molecular graphics. Methods Enzymol. 277, 505-524.

\section{Appendix \\ Free Energy Profile Estimate from Non- equilibrium Trajectories}

To estimate a proton free energy profile from the set of 48 proton trajectories, a maximum likelihood approach was developed. Such an approach was considered necessary due to the non-equilibrium character of the trajectories (cf. Figure 2 of the main text), which precludes the usual approach of calculating the free energy profile from residence frequencies. The method is tailored to optimally reflect the proton dynamics in terms of transitions across the pore, due to both proton transfer events and diffusion, as observed in the collection of trajectories.

For the maximum likelihood calculation, the reaction coordinate (z-axis) was binned in $N 1 \AA$ intervals $i$ between $z=28 \AA$ and $z=80 \AA$ to cover the pore region (see also Figure 2 of the main text), and the z-component of each trajectory was smoothed with a Gaussian filter of $2 \mathrm{fs}$ width. Subsequently, for properly discretized time $t_{i}=j \Delta t$, and for all trajectories, the following numbers of events was counted: $n_{i}^{+}$, the particle is in interval $i$ at time $t_{j}$ and in interval $i+1$ at time $t_{j+1} ; n_{i}^{-}$, the particle is in interval $i$ at time $t_{j}$ and in interval $i-$ 1 at time $t_{j+1} ; n_{i}^{0}$, the particle is in interval $i$ at time $t_{j}$ and stays there at time $t_{j+1}$. The bin index $i$ runs from 0 to $N$ for $n_{i}^{0}$ and formally setting $n_{N}^{+}=0$ and $n_{0}^{-}=0$ will simplify the notations, as it allows all sums to run from $i=0$ to $N$. The time discretization $\Delta t=2 \mathrm{fs}$ was chosen small enough such that jumps over more than one $z$-bin do not occur.

The obtained $3 \mathrm{~N}$ counts characterize each set of trajectories and are used to calculate the conditional probability density $p\left(\left\{n_{i}^{+}, n_{i}^{-}, n_{i}^{0}\right\}_{i=0 \ldots N} \mid\left\{\Delta G_{i}\right\}_{i=0 \ldots N}\right)$ that the trajectory set at hand is observed given a free energy profile $G(z)$. Here, also the free energy profile is discretized along the reaction coordinate defined above, and the differences of adjacent free energy values, $\Delta G_{i}=G_{i+1}-G_{i}$, are used as independent variables that define the free energy profile $G(z)$. We assume that for each time interval $\Delta t$ the probability for a particle within bin $i$ to jump to bin $i+1$ is given by the Boltzmann factor and is proportional to $\Delta t$ :

$$
P_{i \rightarrow i+1} \propto \Delta t \mathrm{e}^{-\beta \Delta G_{i}}
$$

and similarly for jumps from bin $i$ to $i-1$ :

$$
P_{i \rightarrow i-1} \propto \Delta t \mathrm{e}^{+\beta \Delta G_{i-1}}
$$

where $\beta=1 / k_{\mathrm{B}} T$ is the reciprocal thermal energy. After proper normalization one obtains:

$$
\left.\begin{array}{rl}
P_{i \rightarrow i+1} & =\frac{\omega \Delta t \mathrm{e}^{-\beta \Delta G_{i}}}{\omega \Delta t \mathrm{e}^{-\beta \Delta G_{i}}+\omega \Delta t \mathrm{e}^{\beta \Delta G_{i-1}}+1} \\
P_{i \rightarrow i-1} & =\frac{\omega \Delta t \mathrm{e}^{\beta \Delta G_{i-1}}}{\omega \Delta t \mathrm{e}^{-\beta \Delta G_{i}}+\omega \Delta t \mathrm{e}^{\beta \Delta G_{i-1}}+1} \\
P_{i \rightarrow i} & =\frac{1}{\omega \Delta t \mathrm{e}^{-\beta \Delta G_{i}}+\omega \Delta t \mathrm{e}^{\beta \Delta G_{i-1}}+1}
\end{array}\right\}
$$


where the latter equation denotes the probability to stay within reaction coordinate interval $i$ and during the time interval $\Delta t$, and $\omega$ is the (unknown) proportionality factor that may be interpreted as a Kramers' pre-factor or an attempt frequency.

Assuming that all observed jumps are statistically independent, the conditional probability to observe a given set of trajectories for given $\omega$ :

$$
\begin{aligned}
& P\left(\left\{n_{i}^{+}, n_{i}^{-}, n_{i}^{0}\right\}_{i=0 \ldots N} \mid\left\{\Delta G_{i}\right\}_{i=0 \ldots N}, \omega\right) \\
& =\prod_{i=0}^{N} P_{i \rightarrow i+1}^{n_{i}^{+}} P_{i \rightarrow i-1}^{n_{i}^{-}} P_{i \rightarrow i}^{n_{i}^{0}} \\
& =(\omega \Delta t)^{\left(N^{+}+N^{-}\right)} \frac{\exp \left(\beta \sum_{i=0}^{N} n_{i}^{-} \Delta G_{i-1}-\beta \sum_{i=0}^{N} n_{i}^{+} \Delta G_{i}\right)}{\prod_{i=0}^{N}\left(\omega \Delta t \mathrm{e}^{-\beta \Delta G_{i}}+\omega \Delta t e^{\beta \Delta G_{i-1}}+1\right)^{n_{i}}}
\end{aligned}
$$

with $N^{+}=\sum_{i=0}^{N} n_{i}^{+}, \quad N^{-}=\sum_{i=0}^{N} n_{i}^{-}, \quad$ and $n_{i}=$ $n_{i}^{+}+n_{i}^{-}+n_{i}^{0}$, is obtained. Note that the above (absolute) probability scales, as must be expected, with $\Delta t$ to the power of the number of observed jumps; this factor is subsequently separated off in order to obtain the appropriate probability density $p(\cdots)$ (denoted by lower case $p$, to distinguish it from the above absolute probability $P(\cdots)$ ), which for small $\Delta t$ is independent of the particular choice of $\Delta t$ and has been introduced at the beginning of this subsection.

Following Bayes' rule, and assuming that all free energy profiles and attempt frequencies have equal a priori probability, the conditional probability density for free energy profiles given the observed trajectories is found to be equal (up to normalization) to the above reverse conditional probability density. Hence, the most probable energy profile can be found by differentiating $p\left(\left\{n_{i}^{+}, n_{i}^{-}, n_{i}^{0}\right\}_{i=0 \ldots N} \mid\left\{\Delta G_{i}\right\}_{i=0 \ldots N}, \omega\right)$ with respect to the $N+1$ independent variables $\Delta G_{i}$ and $\omega$.

It is more convenient, though, to maximize the logarithm of that probability density, which in the limit of small $\omega \Delta t$ yields the gradients:

$$
\begin{aligned}
& \frac{\partial}{\partial \Delta G_{j}} \ln p(\cdots) \\
& =\frac{\partial}{\partial \Delta G_{j}}\left[\left(N^{+}+N^{-}\right) \ln (\omega \Delta t)\right. \\
& \quad+\beta \sum_{i=0}^{N}\left(n_{i}^{-} \Delta G_{i-1}-n_{i}^{+} \Delta G_{i}\right) \\
& \left.\quad-\sum_{i=0}^{N} n_{i} \ln \left(\omega \Delta t \mathrm{e}^{\beta \Delta G_{i-1}}+\omega \Delta t \mathrm{e}^{-\beta \Delta G_{i}}+1\right)\right] \\
& =\beta\left(n_{j+1}^{-}-n_{j}^{+}\right)-\frac{\beta n_{j+1} \omega \Delta t \mathrm{e}^{\beta \Delta G_{j}}}{1+\omega \Delta t\left(\mathrm{e}^{\beta \Delta G_{j}}+\mathrm{e}^{-\beta \Delta G_{j+1}}\right)} \\
& \quad+\frac{\beta n_{j} \omega \Delta t \mathrm{e}^{-\beta \Delta G_{j}}}{1+\omega \Delta t\left(\mathrm{e}^{\beta \Delta G_{j-1}}+\mathrm{e}^{-\beta \Delta G_{j}}\right)}
\end{aligned}
$$

and, respectively:

$$
\begin{aligned}
\frac{\partial}{\partial \omega} \ln p(\cdots)= & \frac{1}{\omega}\left(N^{+}+N^{-}\right) \\
& -\Delta t \sum_{i=0}^{N} n_{i}\left(\mathrm{e}^{\beta \Delta G_{i-1}}+\mathrm{e}^{-\beta \Delta G_{i}}\right) .
\end{aligned}
$$

Using these gradients, and including a uniform correction term to fulfil the constraint of equal free energy levels at both sides far from the barrier (i.e. in bulk water), the $\Delta G_{i}$ and $\omega$ with largest probability have been computed by steepest ascent.

Note that the obtained profile does not necessarily reflect the detailed microscopic character of the underlying dynamics that occur in the individual trajectories (e.g. the instantaneous proton translocation during a hop event), but rather captures the ensemble behaviour of the collection of trajectories, independent of the underlying microscpoic mechanism. Hence, we refer to the obtained profile as an effective free energy profile.

Edited by G. von Heijne 